\title{
Ontological Modeling of the State Economic Development Policy for Cultural Industries
}

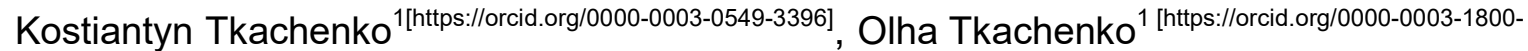 \\ 618X] , Oleksandr Tkachenko 2 [https://orcid.org/0000-0001-6911-2770], Mariia Proskurina 3 [https://orcid.org/0000-0002- \\ 7701-9784], Iryna Parkhomenko 3[https://orcid.org/0000-0002-8328-6774] \\ ${ }^{1}$ State University of Infrastructure and Technology, Ukraine \\ ${ }^{2}$ National Aviation University, Ukraine \\ ${ }^{3}$ Kyiv University of Culture, Ukraine
}

\begin{abstract}
The article discusses an ontological approach to solving the problem of forming state policy of economic development of cultural and creative industries and the corresponding intellectual-information management systems. The purpose of this article is to develop an effective toolkit (based on ontologies) for making optimal decisions in the field of state regulation of the cultural and creative industries, taking into account the dynamic factors of the external environment. The ontological approach considered in the article assumes the presence of three levels of models: meta-ontology, models of subject areas of cultural and creative industries and models of making appropriate management decisions on the formation of economic development policy of cultural and creative industries. The novelty of the proposed approach lies in the purposeful nature of ontological modeling of such complex system as the state policy of economic development of cultural and creative industries. The system under consideration has certain goals, tasks, resources, processes, factors of influence, risks and other characteristics. These characteristics include, in particular, the structure of the model, the ability to highlight the essential objects of real relations of the considered subject areas, the ability to represent knowledge for the joint work of specialists in computer modeling, the processing of expert knowledge and the generation of management decisions within the framework of the corresponding intellectual-information systems.
\end{abstract}

Keywords: Ontological modeling, domain, information system, scenario design, cultural and creative industries

\section{Introduction}

One of the most important tasks of supporting decision-making (managerial and political) in the field of state policy for the development of cultural industries is the task of determining the set of acceptable alternatives for the implementation of this policy, followed by the possibility of choosing the best solution according to the specified optimality criteria.

There are a lot of models (analytical, optimization) of control processes for the functioning and development of complex systems [1], but for solving the assigned tasks (forming a state policy for the development of cultural industries), their use is ineffective due, first of all, to the scale and complexity of the subject area (domain) under consideration.

The use of the concept "industry" in the context of culture determines the orientation of public policy measures primarily on the economic component of the sector. It is also 
advisable to consider cultural and creative industries $(\mathrm{CCl})$ together as a single sector of socio-economic activity. This sector is characterized as multisystem and dynamism of its development, as well as the complexity of interdisciplinary connections.

The state policy for the development of cultural industries involves the management of institutions and the formation of information and cultural space. Mandatory elements of $\mathrm{CCl}$ public administration are [2]:

- $\quad$ setting policy priorities;

- sources of financing for the development of $\mathrm{CCl}$;

- management principles (management codex);

- building an optimal and efficient system for managing subindustries, considering the regional specifics.

Among the main directions of the state policy implementation for the development of $\mathrm{CCl}$, it is possible to single out the following:

- creation of an internal market, which forms the information and cultural space of the country;

- development of the creative class (this requires reforms in the education system and labor legislation);

- creation of an appropriate business climate for the development of creative entrepreneurship (creation of optimal conditions for the commercialization of talent and ideas, favorable tax regime, protection of the producer of a national cultural product, etc.).

The economic development of the sector of cultural and creative industries, being an object of state policy, requires the creation of an adequate model for determining the strategy and tactics of subject area development, making appropriate management decisions and carrying out forecast calculations. Such a model should take into account the complexity, multidimensionality, many goals of economic development of the subject area under consideration, factors of influence, etc.

Semantic or ontological models should be used to display the whole complex of $\mathrm{CCl}$ problems and their economic development.

The purpose of this study is to develop an effective toolkit (based on ontologies) for making optimal decisions in the field of state regulation of $\mathrm{CCl}$, considering the dynamic factors of the external environment.

The existing restrictive conditions impose rather strict requirements on the generated set of alternatives (options) of management decisions. Because of this, the range of admissible alternatives either deliberately narrows or completely excludes the possibility of making an optimal decision (we can only talk about a partially optimal solution).

In practice, this leads to errors of two types: investing in ineffective projects within the framework of the state policy to support the industry, or refusal from more strategically profitable decisions due to an incorrect planning model.

Even if an optimization model is developed and an optimal solution or a set of feasible solutions is obtained for the problem under consideration, then it should be borne in mind that the conditions under which this model and this solution are obtained can change quite quickly.

That is why planning (management) should be considered as a continuous process that allows to adapt public policy (public administration project) to external and internal changing conditions.

At the same time, it should be considered that not every model and not every solution has a sufficient margin of stability so that they can continue to be used without significant adjustment throughout the entire life cycle of the project or its individual stages. 
Management decisions aimed at the economic development of the $\mathrm{CCl}$ should be formed for:

- strategies for the economic development of the $\mathrm{CCl}$ taking into account external influence;

- strategies for economic development of the $\mathrm{CCl}$ taking into account internal conditions;

- creation and promotion of a national cultural product (cultural projects) in domestic and foreign markets;

- resource management (material, financial, legal, personnel, etc.);

- technological support of economic development;

- development of the $\mathrm{CCl}$ infrastructure.

For the subject area under consideration, it is proposed that the generation of managerial decisions is a complex informal process.

To form possible options for management decisions, expert knowledge is required on:

- subject area;

- the current and predicted state of its external environment (factors of influence: external and internal, the hierarchy of the goals of hierarchical development);

- the specifics of the management of CCls, their subsectors, and their economic development.

The problem of finding an expert even for traditional industrial projects is often difficult to solve, and for innovative creative projects, the risks of which are especially high and there is no experience in implementing similar projects, it can become unsolvable.

Experts are not always able to determine all possible scenarios for the development of $\mathrm{CCl}$, and multivariate planning is either not always justified at a sufficient analytical level, or does not have a strategy.

The technology of state policy formation based on the ontological approach can be an addition to the expert knowledge about subject area (domain), the features and possible options for the economic development of subject area [3], [4].

\section{Features of Ontological Modeling of The State $\mathrm{CCl}$ Economic Development Policy}

The implementation of the state policy of $\mathrm{CCl}$ economic development presupposes the presence of a large number of alternative solutions that take into account both the organizational structure of the subject area (the organizational and institutional structure of the state management of culture and $\mathrm{CCl}$ ) and the scenario of its functioning.

Scenario planning is the most common approach to solving problems at the structural level when planning the economic development of $\mathrm{CCl}$, including in public administration [5].

The scenario for the implementation of public policy in the subject area under consideration is a combination of conditions (external and internal) that lead to certain results, to the efficiency and financial feasibility of specific activities and projects or to the creation of a certain creative product.

Before deciding on the feasibility of implementing certain government measures and defining a strategy for public policy in general, possible scenarios should be investigated. This will make it possible to determine the area of sustainability of the subject area to dynamically changing environmental factors and the prospects for the implementation of the policy of support and development of the $\mathrm{CCl}$.

To describe the subject area, it is necessary to define such components as goals, tasks, activities, results and resources. 
Goals are a set (aggregate) of measures of state regulation of the external and internal environment of the $\mathrm{CCl}$ (I, intent).

Tasks are a set of actions (procedures, works and activities) that must be carried out in order to achieve the goals of public policy in the $\mathrm{CCl}$ ( $\mathrm{T}$, task).

Works (operations) are a set of processes aimed at solving problems and obtaining results, requiring the necessary investment of time and resources $(\mathrm{O}$, operation).

Results are a set of political and socio-economic events that embody the goals of politics - these are decisions, creation of institutional structures, implementation of targeted programs, adoption of laws (Rt, result).

Resources are the set of objects needed to get work done (Res). The necessary resources are allocated for the execution of each work.

Thus, the considered domain (D), without taking into account the influence factors, can be represented as:

$$
\mathrm{D}=(\mathrm{I}, \mathrm{T}, \mathrm{O}, \mathrm{Rt}, \mathrm{Res})
$$

Since the state policy is implemented in a certain environment, called the $\mathrm{CCl}$ environment, in addition to describing the domain (see (1)), the scenario includes a description of the external conditions under which the generation of managerial decisions and their implementation is carried out.

The external conditions of implementation (factors of influence) can change quite dynamically throughout the entire life cycle of a complex system, which is the state policy in general, and the policy in the field of $\mathrm{CCl}$, in particular. These factors are sources of possible risks of the implementation of an effective policy to support the economic development of the $\mathrm{CCl}$ and have a direct impact on the process of forming possible options for the corresponding management decisions.

Thus, the considered domain (D) taking into account the external factor of influence (Fe) and internal factor of influence $(\mathrm{Fi})$ can be represented as:

$$
\mathrm{D}=(\mathrm{I}, \mathrm{T}, \mathrm{O}, \mathrm{Rt}, \mathrm{Res}, \mathrm{Fe}, \mathrm{Fi})
$$

When determining the subject area development scenario, all of the above information must be taken into account. As a rule, such information is an unformalized or weakly formalized description, and the complexity of scenario compilation is associated with the need to consider the development of the subject area internal processes and their not always obvious connections with the subject area environment factors and among themselves.

The use of an ontological approach will make it possible to automate the generation of public policy strategies that allow achieving goals taking into account the most important and probable risks. This contributes to a significant increase in objectivity and optimization of the management decision-making process.

Before proceeding with the description of the ontology, it is necessary to determine for what purpose it is being created and what tasks it will solve.

In this work, ontological modeling is used to develop a variety of scenarios for the economic development of the $\mathrm{CCl}$. The proposed ontology should ensure the completeness of coverage of the feasible solutions area to achieve the goals of state policy in the field of culture.

The implementation of the state policy of $\mathrm{CCl}$ economic development is a complex process, which is influenced by many factors; therefore, the formalization of the structural level of multivariate calculations will improve the quality of effective management decisions.

Decisions made at the planning level should be formed with taking into account the possible impact of negative and positive risks of various nature and varying degrees of controllability. 
Implementation of the optimal state policy in the $\mathrm{CCl}$ will minimize the consequences or avoid potential risks for the economic entities of the sector. Among such risks, one should, in particular, highlight:

- geopolitical (threat to the internal information space, cultural intervention, convergence);

- social (creation of semi-public goods, polarization of public opinion, discrimination, lack of qualified personnel);

- technical and technological (lack of modern equipment for the production and consumption of a cultural product, lack of infrastructure, etc.);

- economic (growth in costs for the production of a cultural product, economic instability, inflation, a decrease in consumer solvency, unpredictability of consumer behavior, export-import strategy, tax burden, etc.);

- market (falling product prices, piracy, decrease in sales volumes, increased competition, shadow market, etc.);

- financial (high costs for the production of prototypes, lack of funding for innovative projects, lack of credit programs for startups, high level of financial investment for some industries in the sector, etc.);

- production (quality of a cultural product, creation of a standardized cultural product, copyright protection, etc.);

- political (making ineffective political decisions, lobbying interests, a threat to the decentralization process, lack of specialized legislation in the sector, insufficient level of ensuring the cultural rights of citizens, etc.).

The typification of risks by their levels of manageability (fully manageable, partially managed and unmanaged) contributes to:

- setting priorities in risk management;

- reducing risks in the development of production and financial strategies for the implementation of state policy in the field of $\mathrm{CCl}$;

- the use of information technology and intelligent information systems for making management decisions.

Thus, the toolkit for generating scenarios for the implementation of state policy in the field of culture should have knowledge about:

- available implementation strategies not only at the level of a predefined set of actions;

- the effectiveness of the strategy implementation in accordance with reality;

- reactions to unknown situations (risks, challenges, opportunities) that may arise during the life cycle of the implementation of public policy.

When using the ontological approach, it is important to separate the ontology of the domain from the ontology of problems solved in this domain [6], [7], [8].

This makes it possible to more conveniently describe the dynamic processes of the problems being solved on the basis of static data and knowledge of the domain. Therefore, to ensure the subsequent intellectualization of the generation of options (alternatives) of management decisions, it is advisable to use:

- ontological model of the domain;

- ontological model of the process of constructing an appropriate scenario for the implementation of state policy in the field of culture;

- an ontological model of the process of constructing an appropriate scenario for the economic development of the cultural sphere.

The ontological model proposed by the authors can be represented as $M=\left\langle\mathrm{V}, \mathrm{C}, \mathrm{K}, \mathrm{L}, \mathrm{A}_{l}\right\rangle$, where:

$\mathrm{V}-\mathrm{a}$ set of nodes (primary elements, terms of domain); 
$\mathrm{C}$ - a set of connecting elements of ontological model (of the corresponding ontograph), each of which defines a certain fragment of ontological model (of the ontograph);

$\mathrm{K}$ is the set of key vertices of the ontograph, each of which defines a certain class of equivalent (of the same type) elements of the ontograph $(\mathrm{K} \subset \mathrm{V})$;

$L$ is a set of labels of elements (alphabet of elements) of the ontograph, each of which specifies a certain base class of equivalent elements of the ontograph.

Such classes of elements of ontological model include following classes:

- nodes of the ontograph,

- connecting elements of the ontograph,

- key vertices of the ontograph,

- ontograph labels,

- incidence relations defined on the set of elements of the ontograph.

$A_{l}$ is a set of incidence relations that are defined on a set of ontograph elements. All incidence relationships are binary oriented relationships.

To implement each ontological model, it is necessary to select directly the entities and the relationships between them (knowledge base), as well as the interpretation functions defined for this knowledge base.

The interpretation functions will make it possible to use the created knowledge base for solving various problems of forming the state policy of economic development of the $\mathrm{CCl}$, presented in metaontology and ontologies of a lower level.

The complex of ontological models used to generate management decisions in the formation of the state policy of economic development of the $\mathrm{CCl}$ is presented in the form of a semantic network of ontologies (ontograph) [9].

The ontograph reflects the relationship between the individual components of the cultural industry (its structural and organizational elements, etc.) and is intended for:

- choice of management decisions;

- establishing links between components at the same level and at different levels.

The vertices in the ontograph are various models of the subject area $\mathrm{CCl}$, and the edges are the relations of detailing and decomposition of these models (Figure 1).

The complex of models includes:

- metaontology, as a model of the upper level of generalization;

- ontological model of the domain (cultural and creative industries);

- an applied ontology of the formation procedure (generation) and adoption of managerial decisions (strategic and tactical) in the management systems of $\mathrm{CCl}$ efficiency.

Metaontology is used as a tool for integrating various models of cultural and creative industries and the most general description of the state policy of their economic development. The domain ontological model details some of the top-level metaontology concepts.

The applied ontology of the procedure for the formation and adoption of managerial decisions is intended for the design of appropriate information systems based on ontologies [10]. The complex of models can be extended by various models of the subject area. The model of the subject area - cultural and creative industries - is shown in Figure 1.

This model displays the main directions of the implementation of state policy in the context of the development of the creative economy. 


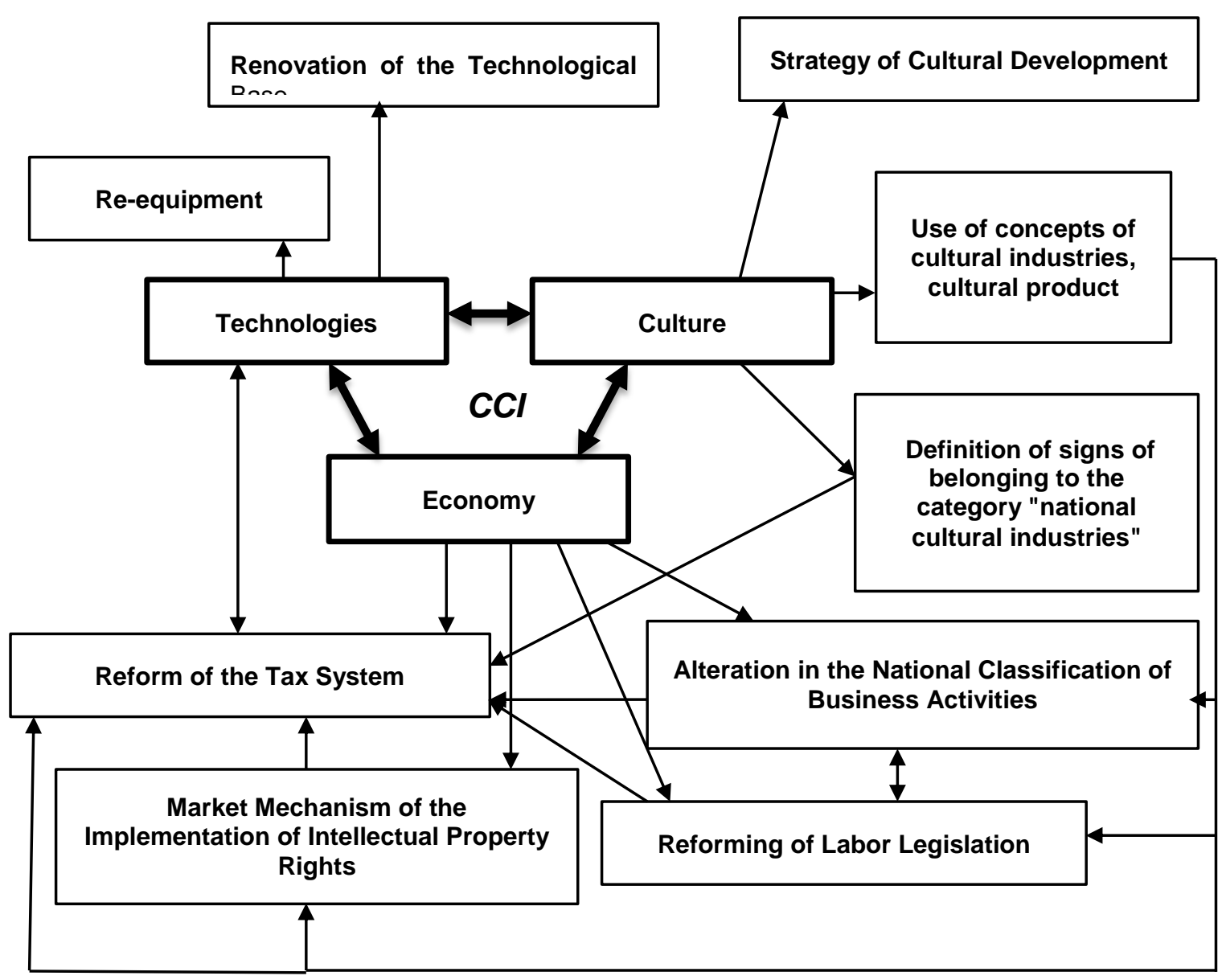

Figure 1. Model of $\mathrm{CCl}$

The ontological model of domain is a formalized description of knowledge about the goals, tasks, works, results and resources of all their cultural projects.

The ontological model is the semantic basis for creating information support for the processes of forming the state policy of economic development of the $\mathrm{CCl}$.

The purpose of the developed ontological model of domain in the context of solving the problem of forming scenarios of state policy in the field of $\mathrm{CCl}$ is to describe the events of the scenario, drawing up a plan for the economic development of $\mathrm{CCl}$, then when constructing an ontological model, the concepts are highlighted based on their influence on the formation of positive and negative financial flows generated by a cultural project.

The questionnaire survey of the Ukrainian $\mathrm{CCl}$ stakeholders (more than 50 representatives of SSH (show business, cinema and theater figures, heads of avent agencies, creative directors of advertising companies, etc.)) made it possible to form a list of significant factors for the implementation of professional activities in the sector and the development of $\mathrm{CCl}$ state policy.

A fragment of the ontograph of the public policy generalized model for the development of the $\mathrm{CCl}$ with an indication of the main concepts for the classes Management, Development and Policy_factures is built in the Protege environment [11], [12] and is shown in Figure 2. 


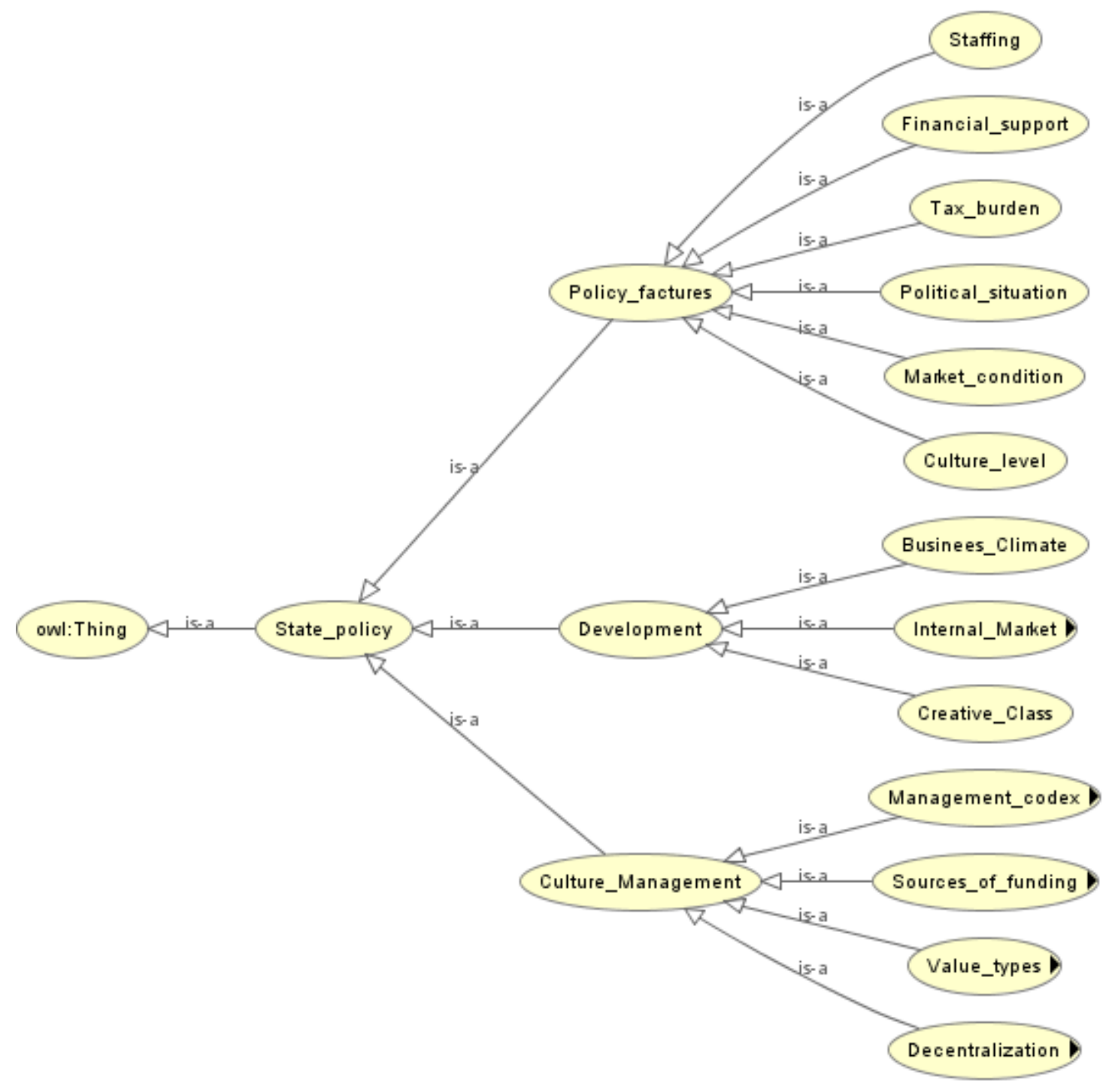

Figure 2. Ontograph for the State_policy class.

A more detailed description of the ontograph for the Development class is shown in Figure 3.

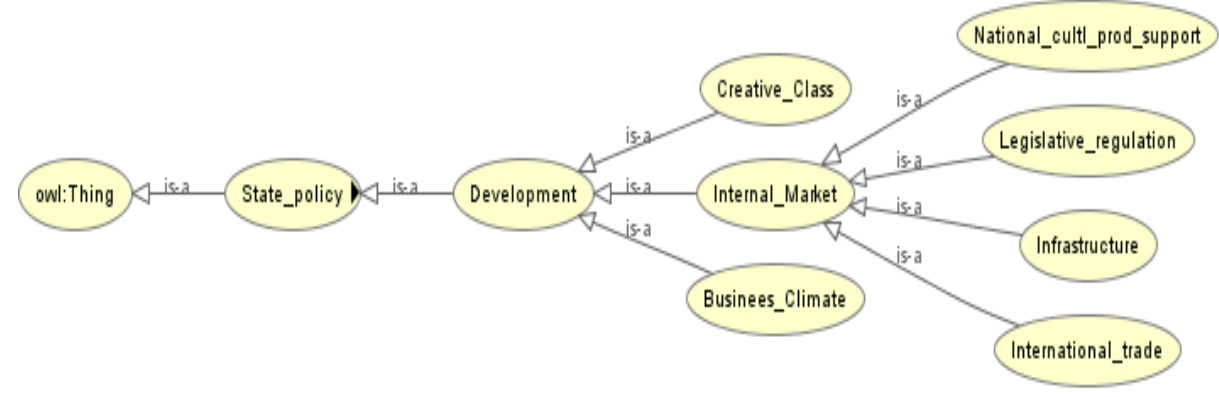

Figure 3. Ontograph for the Development class.

The detailed description of the ontograph for the Culture_Management class is shown in Figure 4. 


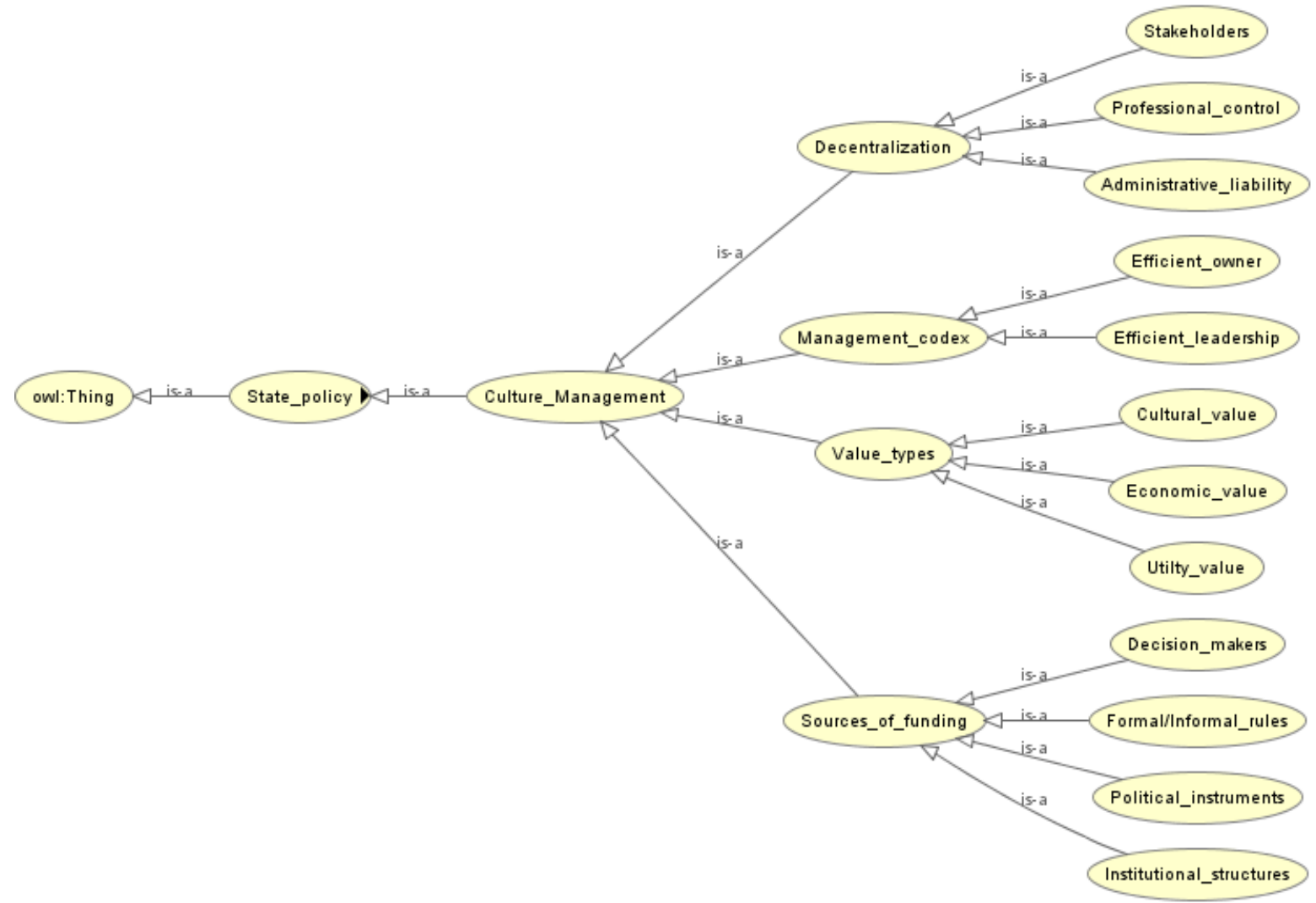

Figure 4. Ontograph for the Culture_Management class.

The structure of the knowledge model based on the proposed ontological approach to modeling should ensure the relationship between:

- alternative strategies for the formation and implementation of the state policy of economic development of the $\mathrm{CCl}$;

- the most possible and probable risks, the negative consequences of which can be minimized through the implementation of a particular strategy;

- characteristics and features of the formation (generation) of control decisions at the current stage of the life cycle of the $\mathrm{CCl}$ project.

The strategic scenario of the state policy for the economic development of the $\mathrm{CCl}$ can be represented by a complex of optimal management decisions in the production, marketing and financial areas of the $\mathrm{CCl}$ project, taking into account the acceptable level of risk, the desired results, the degree of attainability of goals, and the necessary resources.

\section{Formation of Scenarios of the CCI Development}

Creation of scenarios for the formation of the state economic policy of the $\mathrm{CCl}$ development takes into account the planning goals and requires the replenishment of the knowledge base with the necessary information to generate appropriate management decisions.

The degree of scenario detailing directly affects the detail of the subsequent financial calculations for the $\mathrm{CCl}$ project and the choice of tools for quantitative substantiation of management decisions.

When formulating scenarios for the state policy of $\mathrm{CCl}$ economic development at the initial stages of the life cycle, the following is considered:

- the general direction of the formation (planning) of the $\mathrm{CCl}$ development;

- duration of the $\mathrm{CCl}$ project investment stage;

- the duration of the practical implementation of management decisions in the $\mathrm{CCl}$; 
- the term for obtaining results from the implementation of the adopted decisions.

Generation of management decisions that lead to adaptive transformation of scenarios uses:

- a formalized description of scenarios for the formation of the state policy of economic development of the $\mathrm{CCl}$;

- knowledge about the subject area and its environment, accumulated in the knowledge base of the corresponding intelligent information system;

- reasonable forecasts of the influence of potential risk factors (external and internal).

The proposed approach to the formation of the state policy of the $\mathrm{CCl}$ economic development on the basis of ontological modeling is built on the following principles.

- formation of scenarios (their decomposition and synthesis) is performed with taking into account the following:

- the logic of the implementation processes of state support for the development of $\mathrm{CCl}$;

- political, managerial and other aspects of the $\mathrm{CCl}$ project based on the corresponding ontological model;

- variety of scenarios should be based on observed and / or predicted cultural trends,

- content of the scenarios varies depending on the stage of implementation of the state policy for the development of the $\mathrm{CCl}$.

The proposed technology, on the one hand, forms a framework for full discrete coverage of the area of permissible management decisions, and on the other hand it allows the scenarios to be flexibly filled with events with taking into account the required planning detail, as well as taking into account the assumptions of the $\mathrm{CCl}$ project authors and the decision maker (in their role can be customers and the state).

The use of ontological modeling is effective when negotiating between stakeholders and participants in the $\mathrm{CCl}$ project, for example:

- project manager - resource providers (in the formation of managerial decisions and conditions for cooperation to ensure strategies for the state policy of economic development of the $\mathrm{CCl}$ );

- project manager - potential producers of a cultural product, information agencies (in the formation of management decisions and conditions for cooperation to ensure information strategies);

- project manager - potential investors, patrons, sponsors (when forming managerial decisions and terms of cooperation to ensure project financing).

The work did not provide for the use of Wikidata, which may not always fully reflect some specific features of $\mathrm{CCl}$.

\section{Conclusion}

The unified approach to the generation of management decisions and the corresponding intelligent information system has been developed on the basis of ontological modeling of the processes that form the state policy of $\mathrm{CCl}$ economic development.

Using ontological modeling and an appropriate knowledge base, it is possible to generate management decisions at any stage of the life cycle of a cultural project.

Ontographs of various levels of generalization are formed in ontological modeling metaontology, domain ontology and applied ontology for generating the corresponding management decisions.

Metaontology is based on classes of concepts, a system of goals and strategies, organizational and functional structures, a system of criteria for the effectiveness of state policy of economic development of the $\mathrm{CCl}$. 
The ontological model of the subject area can be applied at such stages as the development and formalization of the state policy strategy of the $\mathrm{CCl}$ economic development, the formation of the corresponding scenarios for the $\mathrm{CCl}$ functioning and development.

\section{References}

1. M.R. Kogalovsky, L.A. Kalinichenko, "Conceptual and ontological modeling in information systems", Programming and Computer Software, vol. 35, pp. 241-256, 2009, doi: https://doi.org/10.1134/S0361768809050016

2. A. C. Pratt, T. Hutton, "Reconceptualising the relationship between the creative economy and the recession: learning from the financial crisis. Cities: Asian-European Perspectives", Heidelberg: Springer. 2013, pp. 86-95, doi: https://doi.org/10.1016/j.cities.2012.05.008.

3. F. Neuhaus, Otto von-Guericke, S. Ray, Ram D. Sriram, "Toward Ontology Evaluation across the Life Cycle". nvlpubs.nist.gov. https://nvlpubs.nist.gov/nistpubs/ir/2014/NIST.IR.8008.pdf (accessed Feb.1, 2021). doi: 10.6028/NIST.IR.8008

4. E.M. Sanfilippo, "Feature-based product modelling: an ontological approach", International Journal of Computer Integrated Manufacturing, vol. 31(11), pp. 10971110, 2018, doi: https://doi.org/10.1080/0951192X.2018.1497814.

5. C. List, "Levels: descriptive, explanatory, and ontological". Ise.ac.uk. http://eprints.Ise.ac.uk/87591/1/List_Levels\%20descriptive_2018.pdf (accessed Feb.1, 2021), doi: https://doi.org/10.1111/nous.12241

6. R. Ali, D. Luther, "Scenario Planning: Strategy, Steps and Practical Examples". netsuite.com. https://www.netsuite.com/portal/business-benchmarkbrainyard/industries/articles/cfo-central/scenario-planning.shtml (accessed Feb.1, 2021).

7. K. Munir, "The use of ontologies for effective knowledge modelling and information retrieval". Applied Computing and Informatics, 14(2), pp. 116-126, 2018. https://doi.org/10.1016/j.aci.2017.07.003.

8. O. Tkachenko, A. Tkachenko, K. Tkachenko, "Ontological Modeling of Situational Management", Digital platform: information technology in the sociocultural area, vol. 3. № 1, pp. 22-32, 2020, doi: 10.31866/2617-796x.3.1.2020.206096.

9. A. Gelfert, "The Ontology of Models", in Magnani L., Bertolotti T. (eds) Springer Handbook of Model-Based Science, Springer Handbooks, Springer, Cham, 2017.

10. S.E. Greger, S.V. Porshnev, "Building an ontology of information system architecture". Fundamental Research, № 10, pp. 2405-2409, 2013.

11. "Protégé 5.5". protege.stanford.edu. https://protege.stanford.edu/products.php accessed Feb.1, 2021).

12. M.A. Musen. "The Protégé project: A look back and a look forward Al Matters". Association of Computing Machinery Specific Interest Group in Artificial Intelligence, 1(4), 2015, 10.1145/2757001.2757003. 